\title{
Nanosecond and femtosecond lasers based on black arsenic-phosphorus alloys saturable absorber
}

\author{
Shengjun Huang (黄圣钧) $)^{1}$, Yiran Wang (王神然 $)^{2}$, Jingliang He (何京良 $)^{3}$, Xiancui Su (苏现翠 $)^{2^{*}}$, and Jie Liu (刘 杰) $)^{1^{* *}}$ \\ ${ }^{1}$ Shandong Provincial Engineering and Technical Center of Light Manipulations \& Shandong Provincial Key Laboratory of Optics and Photonic Device, School \\ of Physics and Electronics, Shandong Normal University, Jinan 250358, China \\ ${ }^{2}$ Shandong Key Laboratory of Optical Communication Science and Technology, School of Physics Science and Information Technology, Liaocheng University, \\ Liaocheng 252059, China \\ 3 State Key Laboratory of Crystal Materials, Institute of Novel Semiconductors, Shandong University, Jinan 250100, China \\ *Corresponding author: suxiancui@|cu.edu.cn \\ ${ }^{* *}$ Corresponding author: jieliu@sdnu.edu.cn \\ Received October 28, 2021 | Accepted December 2, 2021 | Posted Online December 23, 2021
}

\begin{abstract}
In this paper, the high-repetition-rate passively Q-switched (PQS) and the femtosecond continuous-wave mode-locked (CWML) lasers are successfully obtained with 2D black arsenic-phosphorus (b-AsP) nanosheets as saturable absorber (SA) at $1 \mu \mathrm{m}$ for the first time, to the best of our knowledge. The saturable absorption properties and ultrafast carrier dynamics of the 2D b-AsP SA are explored by Z-scan and pump-probe techniques. Moreover, according to the measurement of desired nonlinear optical characteristics of the relaxation time of $27 \mathrm{ps}$ and the modulation depth of $7.14 \%$, the PQS and CWML lasers are demonstrated with the highest repetition rate of $2.26 \mathrm{MHz}$ in the PQS laser and the pulse width of $470 \mathrm{fs}$ in the CWML laser. The results show 2D b-AsP SA has enormous potential for pulse modulation in solid-state bulk lasers.
\end{abstract}

Keywords: ultrafast lasers; mode-locked lasers; passively Q-switched lasers; nonlinearity.

DOI: 10.3788/COL202220.021408

\section{Introduction}

After the first, to the best of our knowledge, discovery of graphene in 2004, two-dimensional (2D) nanomaterials have attracted extensive attention due to the quantum confinement effect on the unique mechanical, optical, and electronic properties $^{[1-11]}$. After graphene, many $2 \mathrm{D}$ nanomaterials have sprung up, like transition metal dichalcogenides (TMDs), topological insulators, and black phosphorus (BP), covering an extensive electromagnetic spectral range including insulators, semiconductors, semimetals, and metals ${ }^{[12-14]}$. Among these 2D nanomaterials, BP has a widely tunable band gap with different layers from $2.2 \mathrm{eV}$ in monolayer to $0.3 \mathrm{eV}$ in its bulk form, high electron mobility, and excellently strong in-plane anisotropic physical properties ${ }^{[15-17]}$. It has been widely applied in optoelectronic devices, energy conversion, medicine, and other fields ${ }^{[18-25]}$. However, BP is easy to oxidize in air, which significantly limits its further applications ${ }^{[26]}$.

One effective way to improve the properties of $2 \mathrm{D}$ materials is alloying or doping, which has been demonstrated in a variety of materials like graphene, boron nitride (h-BN), and TMDs ${ }^{[27-31]}$. In 1994, Shirotani et al. reported the superconducting properties of the alloys with synthesizing BP and phosphorus-arsenic alloys firstly, to the best of our knowledge ${ }^{[32]}$. In 2015, Liu et al. demonstrated the characteristic of layered 2D black arsenicphosphorus (b-AsP), where the layered b-AsP materials were semiconductors with tunable band gaps from $0.3 \mathrm{eV}$ down to $0.15 \mathrm{eV}$ via tuning the chemical compositions during material synthesis ${ }^{[33]}$. Until now, b-AsP has been widely researched in electronic and optoelectronic devices due to its unique photoelectric and anisotropic properties and its better air stability compared with $\mathrm{BP}^{[34-37]}$. Nevertheless, the nonlinear optical properties of $2 \mathrm{D}$ b-AsP have not been reported, especially the saturable absorption properties applied to solid-state bulk lasers.

In this paper, a high-quality $2 \mathrm{D}$ b-AsP saturable absorber (SA) is elaborated. Meanwhile, the saturable absorption and ultrafast carrier dynamics properties are explored by the Z-scan and the pump-probe techniques. Furthermore, the passively $Q$-switched (PQS) and the continuous-wave mode-locked (CWML) solidstate bulk lasers operating at $1.0 \mu \mathrm{m}$ are achieved based on the b-AsP SA. The findings illustrate that $2 \mathrm{D}$ b-AsP SA could potentially be nonlinear optical modulators in solid-state bulk lasers.

\section{Results and Discussion}

By using the top-down liquid phase exfoliation (LPE) method, the $2 \mathrm{D} \mathrm{b}$-AsP SA is fabricated. The atomic ratio of As to $\mathrm{P}$ in the 
(a)

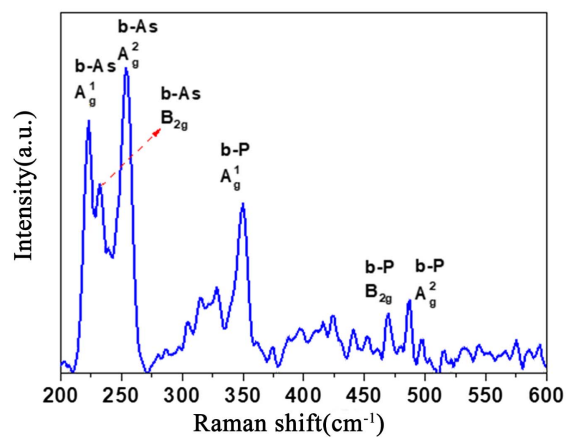

(b)
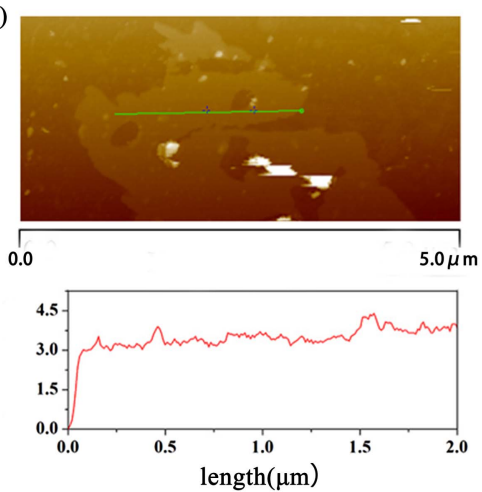

Fig. 1. (a) Raman spectrum of b-AsP nanosheets. (b) AFM image and representative height profile of b-AsP nanosheets.

high-purity commercial b-AsP crystals is approximately 41:9 (the corresponding bandgap is approximately $0.16 \mathrm{eV})^{[33]}$. The surface morphology and structure of the prepared b-AsP SA are researched by the Raman spectra and atomic force microscopy (AFM).

Figure 1(a) presents the unique Raman spectra of b-AsP nanosheets stimulated by a $532 \mathrm{~nm}$ laser at room temperature. The Raman peaks are constituted by the out-of-plane $A_{g}^{1}$ mode, in-plane $B_{g}^{2}$ mode, and out-of-plane $A_{g}^{2}$ mode of b-As, which locate at $223.2,233.9$, and $253.6 \mathrm{~cm}^{-1}$ respectively, while the out-of-plane $\mathrm{A}_{\mathrm{g}}^{1}$ mode, in-plane $\mathrm{B}_{\mathrm{g}}^{2}$ mode, and $\mathrm{A}_{\mathrm{g}}^{2}$ mode of $\mathrm{BP}$ are located at $350.0,467.9$, and $485.6 \mathrm{~cm}^{-1}$, respectively ${ }^{[33]}$. Figure 1(b) shows the AFM image, where the dimensions of the prepared $2 \mathrm{D}$ b-AsP nanosheets are $3 \mu \mathrm{m} \times 4 \mu \mathrm{m}$ with $\sim 4 \mathrm{~nm}$ thickness. The corresponding layer number of $2 \mathrm{D}$ b-AsP nanosheets is about 4-5.

The Z-scan and pump-probe techniques are used to characterize the saturable absorption and the ultrafast carrier dynamics properties of the prepared b-AsP SA. Firstly, the saturable absorption properties of the b-AsP SA are measured by the $\mathrm{Z}$-scan technique through using a fiber laser as the measured light with the repetition rate of $200 \mathrm{kHz}$ and pulse duration of $\sim 15 \mathrm{ps}^{[38]}$. The experimental data is fitted by the following formula ${ }^{[39]}$ :

$$
T=A \times \exp \left(-\frac{\Delta R}{1+\frac{F}{F_{\text {sat }}}}\right)
$$

where $T, A, \Delta R, F$, and $F_{\text {sat }}$ refer to the transmission rate, normalization constant, modulation depth, input fluence, and saturation fluence, respectively. As shown in Fig. 2(a), based on the measured data, the $\Delta R$ of the b-AsP SA is simulated to be $7.14 \%$, while the $F_{\text {sat }}$ is $5.86 \mu \mathrm{J} / \mathrm{cm}^{2}$.

Furthermore, the ultrafast carrier relaxation process of the prepared b-AsP SA is explored by a non-degenerate pumpprobe technique with a femtosecond resolution. The probe wavelength and pump wavelength are $1 \mu \mathrm{m}$ and $800 \mathrm{~nm}$, respectively. The results are shown in Fig. 2(b), in which the experimental data is fitted by an exponential decay function ${ }^{[40]}$ :

$$
\frac{\Delta T}{T_{0}}=A_{1}+A_{2} \times \exp \left(-\frac{t}{\tau}\right)
$$

where $T_{0}, \Delta T, A_{1}, A_{2}$, and $\tau$ refer to the transmittance of the probe laser beam before pump laser excitation, probe laser
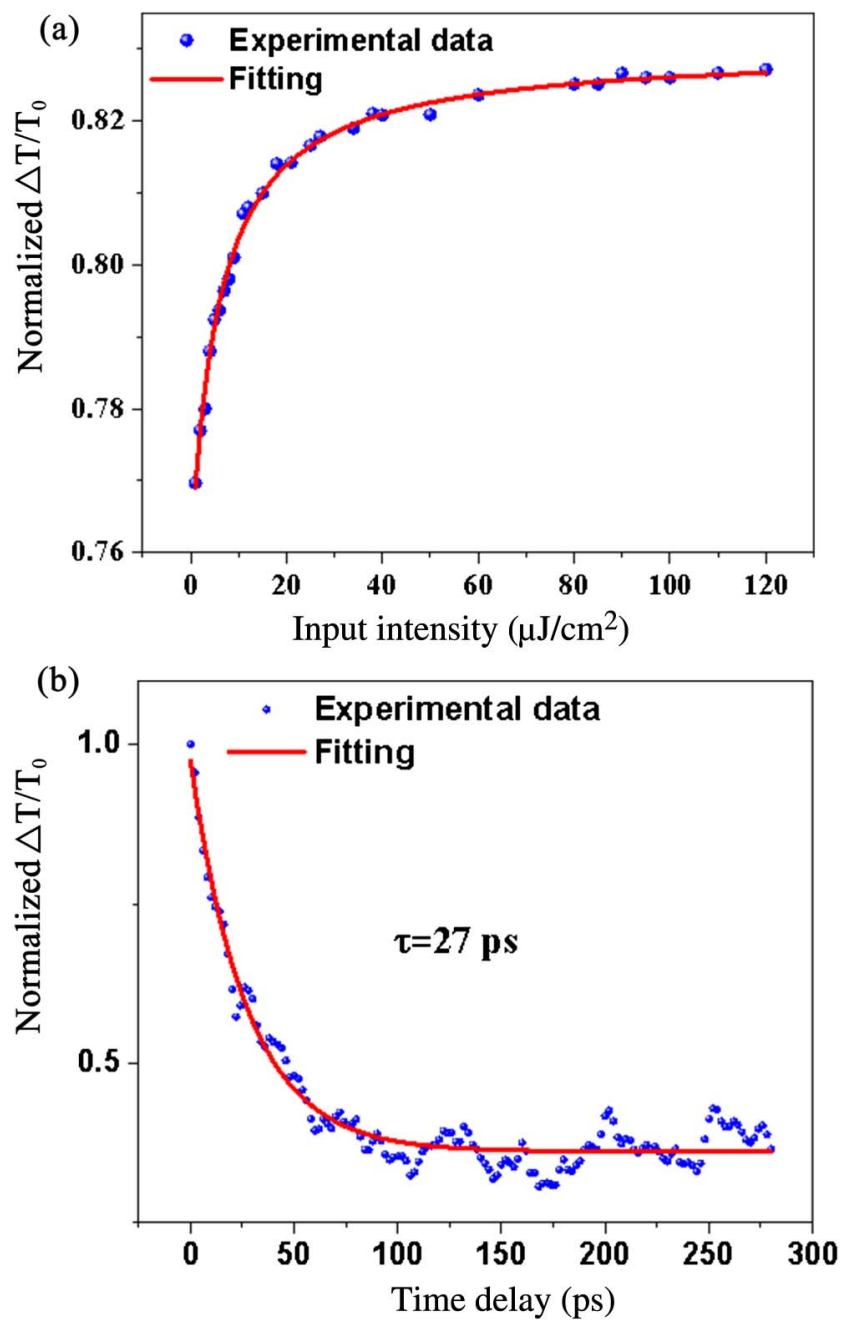

Fig. 2. (a) Transmittance versus incident optical power intensity of b-AsP SA. (b) Time-resolved response of b-AsP SA. 
transmission variation after pump laser excitation, relative amplitudes of the double temporal components, and the intra-band relaxation time related to electron-electron coupling, respectively. By fitting the measured data, the $\tau$ is determined to be $27 \mathrm{ps}$, indicating that the as-prepared b-AsP SA can be applied for ultrafast photonics devices.

The picosecond relaxation time, small saturation fluence, and large modulation depth demonstrate that the prepared b-AsP SA is a slow SA and can be used in the PQS and CWML lasers. Finally, the saturable absorption properties of the $2 \mathrm{D}$ b-AsP SA are demonstrated by the PQS Nd: $\mathrm{YVO}_{4}$ and CWML Yb:KYW lasers. Figure 3(a) shows the experimental setup of PQS laser, which is a concave-plane cavity with a cavity length of $22 \mathrm{~mm}$. The pump source with the central wavelength of $808 \mathrm{~nm}$ is focused on a $3 \mathrm{~mm} \times 3 \mathrm{~mm} \times 10 \mathrm{~mm} \mathrm{Nd}: \mathrm{YVO}_{4}$ crystal (doping concentrations of $0.3 \%$ atomic fraction). The input mirror $(R=200 \mathrm{~mm})$ is high-reflectivity (HR) coated at $0.95-1.1 \mu \mathrm{m}$ and anti-reflectivity (AR) coated at $0.75-0.9 \mu \mathrm{m}$. Meanwhile, a transmission of $10 \%$ at $1064 \mathrm{~nm}$ is used as the output mirror.

By inserting the b-AsP SA into the laser cavity, a stable PQS laser operation is realized. Figure 3(b) depicts the dependence of output powers on absorbed pump powers. When the absorbed pump power is $5 \mathrm{~W}$, a maximum Q-switched output power of $768 \mathrm{~mW}$ is acquired. As shown in Fig. 3(c), the emission wavelength of PQS operation is located at $1064.4 \mathrm{~nm}$. Compared with the CW operation, the full width at half-maximum (FWHM) is narrow, and the central wavelength is blue-shifted, which may be caused by the high insertion loss of the sapphire-based b-AsP SA.

The variations of the pulse durations and repetition rates versus the absorbed pump power are described in Fig. 3(d). The pulse duration decreases from $900 \mathrm{~ns}$ to $140 \mathrm{~ns}$, and the pulse repetition rates increase from $2.26 \mathrm{MHz}$ to $140 \mathrm{kHz}$. As shown in Fig. 3(e), the highest pulse energy is $0.36 \mu \mathrm{J}$ with the highest peak power of $7.5 \mathrm{~W}$. Figure 3(f) describes the characteristic pulse trains and the shortest pulse profiles with different time scales under the maximum output power:

$$
F_{\text {sat }} \Delta R<\frac{\left(P T_{\mathrm{R}}\right)^{2}}{F_{\text {sat }, \mathrm{L}} A_{\mathrm{eff}, \mathrm{L}} A_{\mathrm{eff}, \mathrm{A}}}=\frac{\left(P T_{\mathrm{R}}\right)^{2} \times m \sigma_{\mathrm{em}, \mathrm{L}} \lambda}{h c \pi \omega_{\mathrm{eff}, \mathrm{L}}^{2} \times \pi \omega_{\mathrm{eff}, \mathrm{A}}^{2}},
$$

where $T_{\mathrm{R}}, P, A_{\text {eff,L }}$, and $A_{\text {eff, } \mathrm{A}}$ refer to round-trip time, laser power in the laser cavity, and laser mode areas on laser crystal and SA, respectively. According to the Eq. (3), the result is that $0.42 \mu \mathrm{J} / \mathrm{cm}^{2}$ on the left is far less than $17.2 \mu \mathrm{J} / \mathrm{cm}^{2}$ on the right. Therefore, stable CWML lasers can be obtained with the b-AsP SA.

The setup of CWML laser is demonstrated in Fig. 4(a), a fibercoupled $976 \mathrm{~nm}$ LD is focused on an Np-cut Yb:KYW with a size of $3 \mathrm{~mm} \times 3 \mathrm{~mm} \times 4 \mathrm{~mm}$. In the laser cavity, a Gires-Tournois interferometer (GTI) mirror with a total of $-500 \mathrm{fs}^{2}$ group delay dispersion (GDD) per round is used to compensate the normal GDD. Figure 4(b) indicates that the pulse width is measured by an autocorrelation and fitted by $\operatorname{sech}^{2}$-pulse shape to be about $470 \mathrm{fs}$. It is noted that the central wavelength is $1048 \mathrm{~nm}$ with the FWHM of $3.8 \mathrm{~nm}$ in Fig. 4(c). Compared with the Fourier transform limit value of 0.315 , the corresponding time-bandwidth product of 0.448 is slightly larger, indicating that a slight chirping exists.

A spectrum analyzer records the radio frequency (RF) in Fig. 4(d). The central peak and signal-to-noise ratio are obviously located at $41.8 \mathrm{MHz}$ and up to $58 \mathrm{~dB}$ under the resolution bandwidth (RBW) of $1 \mathrm{kHz}$. The RF spectra show the immaculate and stable CWML operation. (a)

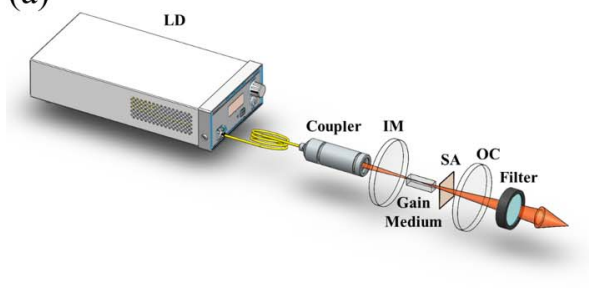

(d)

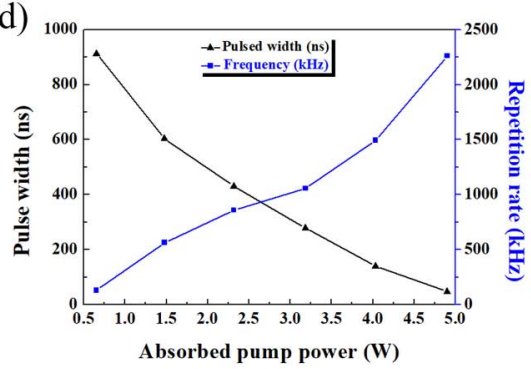

(b)

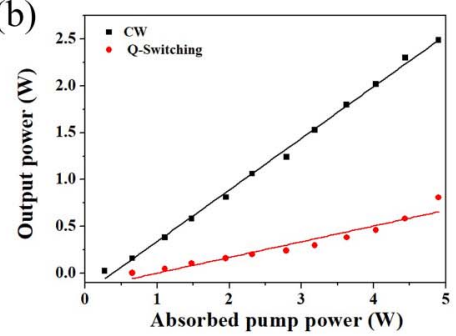

(e)

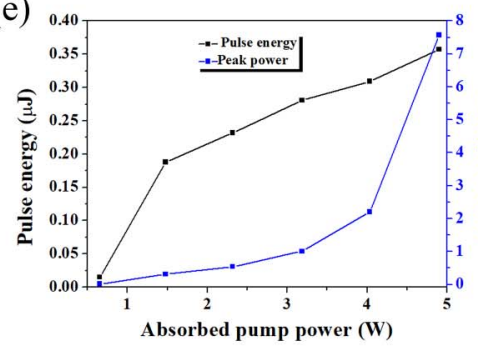

(c)

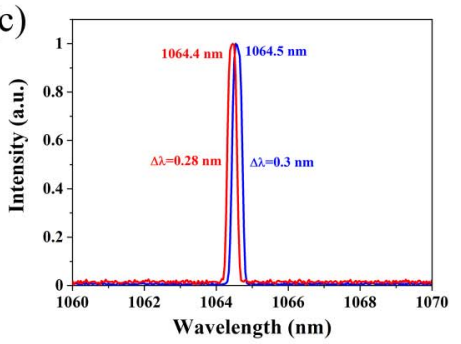

(f)

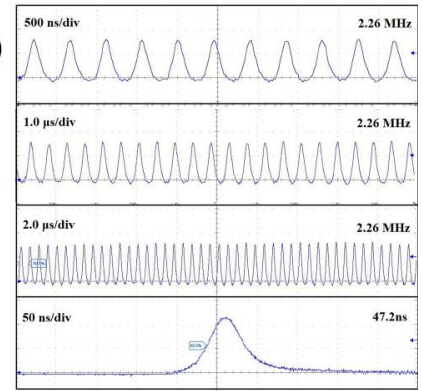

Fig. 3. (a) Experimental setup of PQS. (b) The ratio of output powers to absorbed pump powers under the CW and PQS operations. (c) The emission wavelength of the CW and PQS lasers. (d) Pulse durations and repetition rates versus absorbed pump powers. (e) Pulse energy and peak power versus absorbed pump power. (f) The pulse trains and the shortest pulse profiles with different time scales. 
(a)

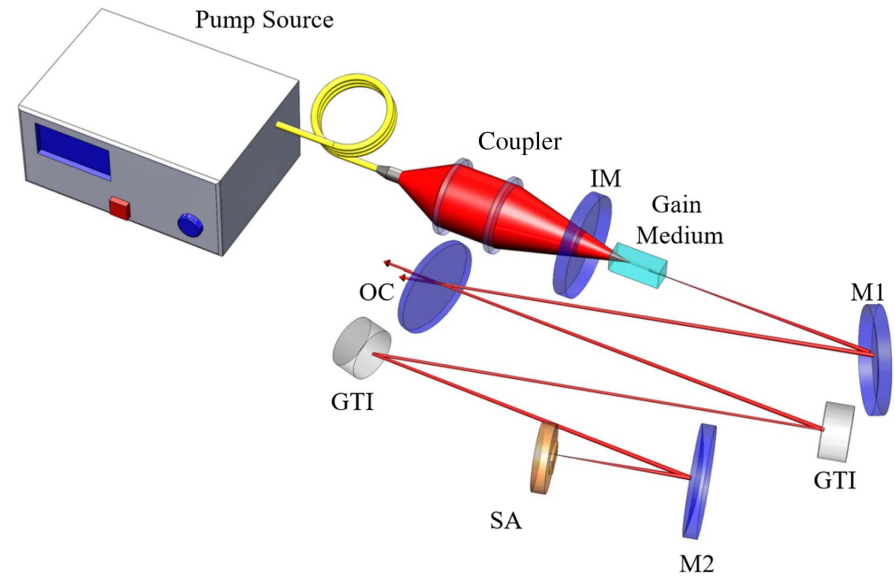

(b)

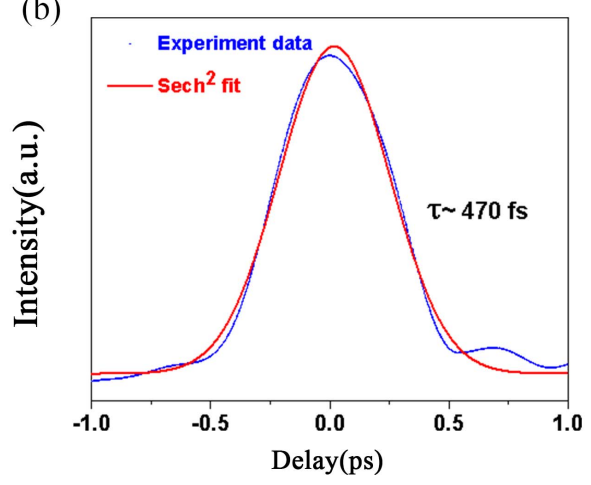

(d)

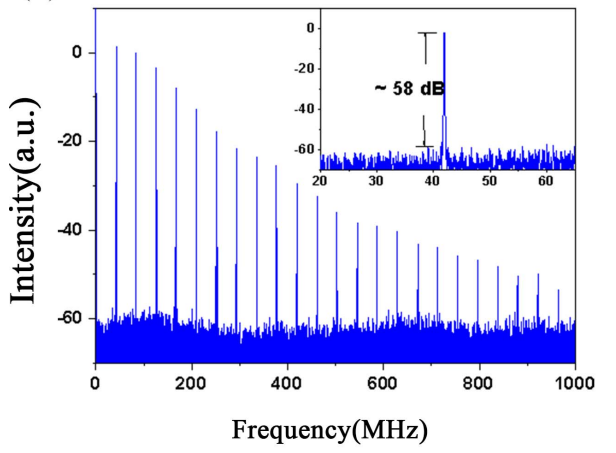

(c)

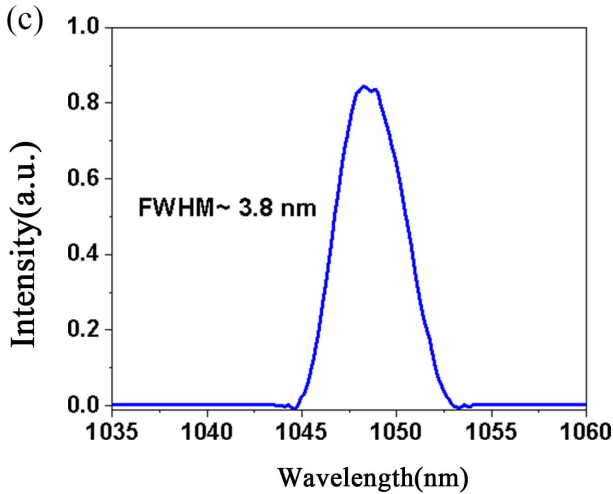

(e)
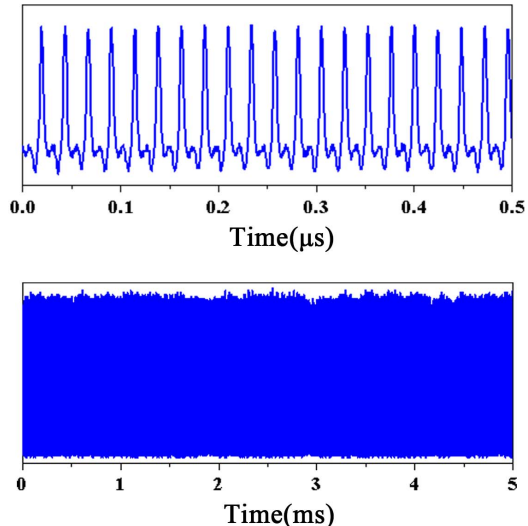

Fig. 4. (a) Experimental setup of CWML laser. (b) CWML laser spectrum. (c) Autocorrelation trace. (d) The frequency spectrum with a wide and narrow span. (e) Pulse trains at the maximum pump power.

The output laser pulse trains with different time scales are presented in Fig. 4(e), which can remain stable over $24 \mathrm{~h}$ in the stability test. Compared with the BP SA applied on the solid-state CWML laser in the previous work ${ }^{[41]}$, although the pulse width of our b-AsP SA is relatively wide, it can still work normally after a month of resting in air, demonstrating the excellent air stability of our sample.

\section{Conclusions}

In conclusion, based on the prepared high-quality b-AsP SA, this paper explores the ultrafast carrier dynamics and saturable absorption properties. Furthermore, saturable absorption properties are experimentally demonstrated by the PQS and CWML lasers. The results show that the highest repetition rate of the PQS laser is $2.26 \mathrm{MHz}$, and the pulse duration of the CWML laser is $470 \mathrm{fs}$, demonstrating that b-AsP SA could be an excellent nonlinear optical modulator for pulse modulation in solidstate bulk lasers.

\section{Acknowledgement}

This work was supported by the National Natural Science Foundation of China (NSFC) (Nos. 11974220 and 61905104). 


\section{References}

1. K. S. Novoselov, A. K. Geim, S. V. Morozov, D. Jiang, Y. Zhang, S. V. Dubonos, I. V. Grigorieva, and A. A. Firsov, "Electric field effect in atomically thin carbon films," Science 306, 666 (2004).

2. Z. Yang, L. Han, Q. Yang, X. Ren, S. Z. U. Din, X. Zhang, J. Leng, J. Zhang, B. Zhang, K. Yang, J. He, C. Li, and J. Wang, "Two-dimensional tellurium saturable absorber for ultrafast solid-state laser," Chin. Opt. Lett. 19, 031401 (2021).

3. Q. Hao, C. Wang, W. Liu, X. Liu, J. Liu, and H. Zhang, "Low-dimensional saturable absorbers for ultrafast photonics in solid-state bulk lasers: status and prospects," Nanophotonics 9, 2603 (2020)

4. X. Feng, J. Liu, W. Yang, X. Yu, S. Jiang, T. Ning, and J. Liu, "Broadband indium tin oxide nanowire arrays as saturable absorbers for solid-state lasers," Opt. Express 28, 1554 (2020).

5. T. Tang, F. Zhang, M. Wang, Z. Wang, and X. Xu, "Two-dimensional tellurene nanosheets as saturable absorber of passively $Q$-switched Nd:YAG solidstate laser," Chin. Opt. Lett. 18, 041403 (2020).

6. B. Guo, " $2 \mathrm{D}$ noncarbon materials-based nonlinear optical devices for ultrafast photonics," Chin. Opt. Lett. 16, 020004 (2018).

7. J. Hong, C. Jin, J. Yuan, and Z. Zhang, "Atomic defects in two-dimensional materials: from single-atom spectroscopy to functionalities in opto-/ electronics, nanomagnetism, and catalysis," Adv. Mater. 29, 1606434 (2017).

8. A. J. Mannix, B. Kiraly, M. C. Hersam, and N. P. Guisinger, "Synthesis and chemistry of elemental 2D materials," Nat. Rev. Chem. 1, 14 (2017).

9. Q. Fu and X. Bao, "Surface chemistry and catalysis confined under twodimensional materials," Chem. Soc. Rev. 46, 1842 (2017).

10. C. Tan, X. Cao, X. Wu, Q. He, J. Yang, X. Zhang, J. Chen, W. Zhao, S. Han, G. Nam, M. Sindoro, and H. Zhang, "Recent advances in ultrathin twodimensional nanomaterials," Chem. Rev. 117, 6225 (2017).

11. Y. Xue, Q. Zhang, W. Wang, H. Cao, Q. Yang, and L. Fu, "Opening twodimensional materials for energy conversion and storage: a concept," Adv. Energy Mater. 7, 1602684 (2017).

12. G. Cunningham, M. Lotya, C. S. Cucinotta, S. Sanvito, S. D. Bergin, R. Menzel, M. S. P. Shaffer, and J. N. Coleman, "Solvent exfoliation of transition metal dichalcogenides: dispersibility of exfoliated nanosheets varies only weakly between compounds," ACS Nano 6, 3468 (2012).

13. Q. H. Wang, K. Kalantar-Zadeh, A. Kis, J. N. Coleman, and M. S. Strano, "Electronics and optoelectronics of two-dimensional transition metal dichalcogenides," Nat. Nanotechnol. 7, 699 (2012).

14. R. Lv, J. A. Robinson, R. E. Schaak, D. Sun, Y. Sun, T. E. Mallouk, and M. Terrones, "Transition metal dichalcogenides and beyond: synthesis, properties, and applications of single- and few-layer nanosheets," Acc. Chem. Res. 48, 56 (2015).

15. F. Xia, H. Wang, and Y. Jia, "Rediscovering black phosphorus as an anisotropic layered material for optoelectronics and electronics," Nat. Commun. 5, 4458 (2014).

16. X. Wang, A. M. Jones, K. L. Seyler, V. Tran, Y. Jia, H. Zhao, H. Wang, L. Yang, $\mathrm{X}$. $\mathrm{Xu}$, and $\mathrm{F}$. Xia, "Highly anisotropic and robust excitons in monolayer black phosphorus," Nat. Nanotechnol. 10, 517 (2015).

17. J. Qiao, X. Kong, Z. Hu, F. Yang, and W. Ji, "High-mobility transport anisotropy and linear dichroism in few-layer black phosphorus," Nat. Commun. 5, 4475 (2014).

18. G. Qu, T. Xia, W. Zhou, X. Zhang, H. Zhang, L. Hu, J. Shi, X. F. Yu, and G. Jiang, "Property-activity relationship of black phosphorus at the nanobio interface: from molecules to organisms," Chem. Rev. 120, 2288 (2020).

19. M. Zhang, Q. Wu, F. Zhang, L. Chen, X. Jin, Y. Hu, Z. Zheng, and H. Zhang, "2D black phosphorus saturable absorbers for ultrafast photonics," Adv. Opt. Mater. 7, 1800224 (2019).

20. Z. He, Y. Zheng, H. Liu, M. Li, H. Lu, H. Zhang, Q. Feng, and D. Mao, "Passively Q-switched cylindrical vector laser based on a black phosphorus saturable absorber," Chin. Opt. Lett. 17, 020004 (2019).

21. B. Deng, R. Frisenda, C. Li, X. Chen, A. Castellanos-Gomez, and F. Xia, "Progress on black phosphorus photonics," Adv. Opt. Mater. 6, 1800365 (2018).

22. Y. Xue, Z. Xie, Z. Ye, X. Hu, J. Xu, and H. Zhang, "Enhanced saturable absorption of $\mathrm{MoS}_{2}$ black phosphorus composite in $2 \mu \mathrm{m}$ passively Q-switched Tm:YAP laser," Chin. Opt. Lett. 16, 020018 (2018).

23. J. Pang, A. Bachmatiuk, Y. Yin, B. Trzebicka, L. Zhao, L. Fu, R. G. Mendes, T. Gemming, Z. Liu, and M. H. Rummeli, "Applications of phosphorene and black phosphorus in energy conversion and storage devices," Adv. Energy Mater. 8, 1702093 (2018).

24. Y. Liu, T. Sun, W. Ma, W. Yu, S. B. Nanjunda, S. Li, and Q. Bao, "Highly responsive broadband black phosphorus photodetectors," Chin. Opt. Lett. 16, 020002 (2018).

25. X. Wang, Z. Wang, Y. Wang, L. Li, G. Yang, and J. Li, "Watt-level high-power passively $Q$-switched laser based on a black phosphorus solution saturable absorber," Chin. Opt. Lett. 15, 011402 (2017).

26. J. O. Island, G. A. Steele, H. S. J. V. Zant, and A. Castellanos-Gomez, "Environmental instability of few-layer black phosphorus," 2D Mater. 2, 11002 (2015).

27. B. Konkena, J. Masa, W. Xia, M. Muhler, and W. Schuhmann, "MoSSe@reduced graphene oxide nanocomposite heterostructures as efficient and stable electrocatalysts for the hydrogen evolution reaction," Nano Energy 29, 46 (2016).

28. Y. Gong, Z. Liu, A. R. Lupini, G. Shi, J. Lin, S. Najmaei, Z. Lin, A. L. Elías, A. Berkdemir, G. You, H. Terrones, M. Terrones, R. Vajtai, S. T. Pantelides, S. J. Pennycook, J. Lou, W. Zhou, and P. M. Ajayan, "Band gap engineering and layer-by-layer mapping of selenium-doped molybdenum disulfide," Nano Lett. 14, 442 (2014).

29. S. Li, Y. Zhai, X. Zhang, and D. R. MacFarlane, "Surfactant-free synthesis of graphene-supported $\mathrm{PdCu}$ nanocrystals with high alloying degree as highly active catalyst for formic acid electrooxidation," Adv. Mater. Interfaces $\mathbf{4}$, 1700227 (2017).

30. M. L. Parucker, C. E. Da Costa, and V. L. Soethe, "Development of Ni/h-BN self-lubricating composite powder by high-energy ball milling," Mater. Sci. Forum 869, 277 (2016).

31. D. Alfè, M. Pozzo, E. Miniussi, S. Günther, P. Lacovig, S. Lizzit, R. Larciprete, B. S. Burgos, T. O. Menteş, A. Locatelli, and A. Baraldi, "Fine tuning of graphene-metal adhesion by surface alloying," Sci. Rep. 3, 2430 (2013).

32. I. I. Shirotani, J. Mikami, T. Adachi, Y. Katayama, K. Tsuji, H. Kawamura, O. Shimomura, and T. Nakajima, "Phase transitions and superconductivity of black phosphorus and phosphorus-arsenic alloys at low temperatures and high pressures," Phys. Rev. B 50, 16274 (1994).

33. B. Liu, M. Köpf, A. N. Abbas, X. Wang, Q. Guo, Y. Jia, F. Xia, R. Weihrich, F. Bachhuber, F. Pielnhofer, H. Wang, R. Dhall, S. B. Cronin, M. Ge, X. Fang, T. Nilges, and C. Zhou, "Black arsenic-phosphorus: layered anisotropic infrared semiconductors with highly tunable compositions and properties," Adv. Mater. 27, 4423 (2015).

34. O. Osters, T. Nilges, F. Bachhuber, F. Pielnhofer, R. Weihrich, M. Schöneich, and P. Schmidt, "Synthesis and identification of metastable compounds: black arsenic-science or fiction?" Angew. Chem. Int. Ed. 51, 2994 (2012).

35. S. Yuan, C. Shen, B. Deng, X. Chen, Q. Guo, Y. Ma, A. Abbas, B. Liu, R. Haiges, C. Ott, T. Nilges, K. Watanabe, T. Taniguchi, O. Sinai, D. Naveh, C. Zhou, and F. Xia, "Air-stable room-temperature mid-infrared photodetectors based on $\mathrm{hBN} / \mathrm{black}$ arsenic phosphorus/hBN heterostructures," Nano Lett. 18, 3172 (2018).

36. M. Long, A. Gao, P. Wang, H. Xia, C. Ott, C. Pan, Y. Fu, E. Liu, X. Chen, W. Lu, T. Nilges, J. Xu, X. Wang, W. Hu, and F. Miao, "Room-temperature high detectivity mid-infrared photodetectors based on black arsenic phosphorus," Sci. Adv. 3, e1700589 (2017).

37. M. Amani, E. Regan, J. Bullock, G. H. Ahn, and A. Javey, "Mid-wave infrared photoconductors based on black phosphorus-arsenic alloys," ACS Nano. 11, 11724 (2017).

38. R. Zhou, P. Tang, Y. Chen, S. Chen, C. Zhao, H. Zhang, and S. Wen, "Largeenergy, narrow-bandwidth laser pulse at $1645 \mathrm{~nm}$ in a diode-pumped Er:YAG solid-state laser passively $Q$-switched by a monolayer graphene saturable absorber," Appl. Opt. 53, 254 (2014).

39. H. Zhang, J. He, Z. Wang, J. Hou, B. Zhang, R. Zhao, K. Han, K. Yang, H. Nie, and X. Sun, "Dual-wavelength, passively Q-switched Tm:YAP laser with black phosphorus saturable absorber," Opt. Mater. Express 6, 2328 (2016).

40. J. Liu, V. Khayrudinov, H. Yang, Y. Sun, B. Matveev, M. Remennyi, K. Yang, T. Haggren, H. Lipsanen, F. Wang, B. Zhang, and J. He, "InAs-nanowirebased broadband ultrafast optical switch," J. Phys. Chem. Lett. 10, 4429 (2019).

41. X. Su, Y. Wang, B. Zhang, R. Zhao, K. Yang, J. He, Q. Hu, Z. Jia, and X. Tao, "Femtosecond solid-state laser based on a few-layered black phosphorus saturable absorber," Opt. Lett. 41, 1945 (2016). 